\section{PSICOLOGÍA IBEROAMERICANA}

\section{Psicología Iberoamericana}

ISSN: 1405-0943

psicología.iberoamericana@uia.mx

Universidad Iberoamericana, Ciudad de

México

México

Padilla Gámez, Nélida; Díaz-Loving, Rolando

El impacto de la cultura y la familia en la elección de pareja: diferencias entre hombres y mujeres

Psicología Iberoamericana, vol. 20, núm. 1, enero-junio, 2012, pp. 9-17

Universidad Iberoamericana, Ciudad de México

Distrito Federal, México

Disponible en: http://www.redalyc.org/articulo.oa?id=133924623002

Cómo citar el artículo

Número completo

- Más información del artículo

Página de la revista en redalyc.org

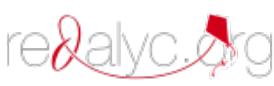

Sistema de Información Científica

Red de Revistas Científicas de América Latina, el Caribe, España y Portugal

Proyecto académico sin fines de lucro, desarrollado bajo la iniciativa de acceso abierto 


\title{
El impacto de la cultura y la familia en la elección de pareja: diferencias entre hombres y mujeres
}

\author{
The Impact of Culture and Family in Mate Choice: Differences \\ between Men and Women
}

\author{
Nélida Padilla Gámez* \\ Rolando Díaz-Loving** \\ Unidad de InVestigaciones Psicosociales, Facultad de Psicología \\ Universidad Nacional Autónoma de México, México
}

\section{RESUMEN}

El presente estudio versa sobre el impacto de la cultura a través de la familia, y en cómo hombres y mujeres eligen pareja. A pesar de que hoy en día se elige al (a) compañero(a) en función de gustos y afinidades personales, en la realidad parece que la familia sigue participando consciente o inconscientemente en la elección de pareja de sus miembros. En épocas pasadas ésta se hacía con base en intereses meramente económicos y familiares; sin embargo, al pasar de los años, esto fue cambiando hasta el punto en que se supone que la elección es totalmente una decisión personal. En este sentido, el objetivo de este estudio fue saber si existen consejos o recomendaciones de la familia y la cultura para elegir pareja, y si son tomados en cuenta para emparejarse. Para llevar a cabo este estudio se aplicó un cuestionario abierto con las preguntas antes mencionadas a 228 participantes, de los cuales 154 eran mujeres y 74 hombres. Las edades fluctuaron entre los 19 y 56 años. Provenían de diversas universidades públicas, privadas y algunos centros de trabajo. Tras hacer un análisis de contenido se identificaron 28 códigos que se agruparon en cuatro familias. A su vez, se realizaron $\mathrm{X}^{2}$ para identificar si existen diferencias por sexo en las categorías. Los resultados indican que más de la mitad de la muestra refiere tomar en cuenta las recomendaciones de su familia, aunado a que existen diferencias en lo que la cultura y la familia recomiendan a hombres y a mujeres.

Descriptores: pareja, elección de pareja, emparejamiento, cultura y familia.

\section{ABSTRACT}

The present study concerns the impact of culture through the family as men and women choose partners. Although today is chosen according couple of tastes and personal affinities, in reality it seems that the family continues to participate consciously o unconsciously in mate choice of members. In the past the choice was made based on purely economic interests and family; as years passed, this changed to the point where it is assumed that the choice is entirely a personal decision. In this sense, the objective of this study was to determine if any advice or recommendations from family and culture to choosing a mate, and if taken into account to match. To carry out this study, a questionnaire was open to the questions above to 228 participants, of which 154 were women and 74 men. The ages ranged between 19 and 56 years. From several public universities, private and some work places. After doing a content analysis identify whether there are sex differences in the categories. The results indicate that more than half of the sample referred to take into account the recommendations of his family coupled with that there are differences in culture and family recommend men and women.

Keywords: partner, mate choice, matching, family and culture.

\footnotetext{
* Para Correspondencia: Mtra. Nélida Padilla Gámez. Unidad de Investigaciones Psicosociales, Facultad de Psicología, unAm. Av. Universidad 3004. Col. Copilco-Universidad. C.P. 04510. Delegación Coyoacán. México D.F. Tel. 5622-2326. e-mail: nelidapg75@yahoo.com.mx

** Dr. Rolando Díaz-Loving. Unidad de Investigaciones Psicosociales, Facultad de Psicología, unam. Av. Universidad 3004. Col. Copilco-Universidad. C.P. 04510. Delegación Coyoacán. México D.F. Tel. 5622-2326. e-mail: rdiazl@unam.mx
} 
La investigación en el tema del emparejamiento ha demostrado que los factores implicados en el proceso mediante el cual dos personas se eligen como pareja son diversos. La perspectiva evolutiva menciona que los hombres eligen mujeres con ciertos rasgos físicos que indiquen salud, juventud y fertilidad. En cambio, ellas elegirán hombres con ciertas características de corte sociocultural, como dinero y estatus (Gangestad, Haselton \& Buss, 2006). No obstante, a la hora de elegir pareja esta perspectiva no siempre opera así. Al parecer, existen otros elementos que influyen en las personas al momento de emparejarse.

En la actualidad se observan múltiples cambios en diversas esferas que atañen al ser humano. Desde la tecnología que avanza a pasos agigantados, hasta el ámbito de las relaciones interpersonales, en donde las opiniones acerca del matrimonio, la familia y la pareja en particular han sufrido una transformación radical (Quilodran, 2001), desde la unión libre, que es cada vez más frecuente, hasta las nuevas tendencias de relación como las familias "Queer" o las parejas LAT (Living Apart Togheter).

Sin embargo, en México, al parecer, todavía se mantienen ciertas premisas y patrones conductuales derivados de lo que la sociocultura establece como adecuado para relacionarse. De esta forma, aunque la sociedad se transforma continuamente, las relaciones interpersonales suelen mantener esos matices tradicionales derivados de lo que familias de origen y extensas dictaminan qué es lo adecuado en la elección de pareja $y$, por ende, en el emparejamiento.

Las actitudes hacia el matrimonio han cambiado; en estos días ya no es visto como la única y mejor opción para vivir en pareja, ya que ésta se forma en un contexto y en una situación en particular (Giddens, 2001), en donde la sociocultura da la pauta al desarrollo de la pareja. Es entonces que las actitudes hacia esta condición se encaminan en dos direcciones: la modernidad y la tradicionalidad.

Así, un aspecto inherente es la influencia que la familia refleja en las ideas y actitudes de la pareja moderna, debido a que cada miembro proviene de un núcleo familiar único. Existen familias que se describen como tradicionales o modernas en mayor o menor medida.

En este sentido, las premisas socioculturales que formula Díaz Guerrero (2003) proporcionan la visuali- zación acerca de cómo algunos mandatos se han transformado pero otros siguen vigentes.

De esta manera, la familia es la base sobre la cual se forma la pareja, la cual, en el futuro, replicará la forma de su familia de origen, tomará algunos aspectos o se constituirá de manera totalmente diferente.

La familia es un importante agente socializador, ya que es a través de esta que se transmiten los roles, papeles, normas, reglas, etc., que dicta la cultura. En este proceso, la información se trasmite de diferentes formas tanto para hombres como para mujeres (Garrido Garduño, Reyes Luna, Torres Velázquez \& Ortega Silva, 2008).

A las mujeres se les educa para buscar hombres exitosos, protectores, trabajadores, estables y buenos proveedores; en cambio a los hombres se les forma para emparejarse con mujeres tradicionales, educadas, buenas madres, amas de casa y que finalmente puedan ser buenas administradoras de sus recursos.

Al respecto, Díaz-Loving, Rocha Sánchez y Rivera Aragón (2007) mencionan que estas diferencias, más que biológicas, provienen de una serie de construcciones socioculturales emanadas de procesos de socialización y endoculturación, que trazan la línea que debería seguir cada sexo, estableciendo y propiciando comportamientos, actividades, preferencias, gustos y deseos diferentes.

De tal forma se supone que estas diferencias impactan en la manera en que se comportan hombres y mujeres; es decir, los hombres se asumen como trabajadores, responsables, inteligentes, fuertes y realizan actividades productivas y públicas, en tanto las mujeres se perciben como cariñosas, amorosas, maternales, comprensivas y se dedican al proceso de crianza y cuidado de los hijos y la familia.

Sin embargo, lainvestigación psicológica ha demostrado que la mayoría de las diferencias son relativas y tienen que ver con la forma de pensar, hacer, sentir, etc., de una sociocultura particular. En este sentido, las diferencias biológicas producen estereotipos y patrones de socialización que son traducidas en diferencias conductuales y sociales.

Esta diferenciación ha impactado en diversos aspectos, ya que se favorece la desigualdad social entre los sexos en función de la posesión de ciertos atributos vinculados a una u otra dimensión, al mismo tiempo 
que encajona a hombres y mujeres en moldes que impiden su cabal desarrollo como seres humanos íntegros.

Se supone que estos patrones tradicionales se están transformando con el paso del tiempo, dada la inserción de las mujeres en el ámbito laboral y académico, lo cual ha permitido la adquisición y desarrollo de características tradicionalmente bosquejadas como "masculinas". De igual forma, aunque en menor medida, se observa que los hombres han empezado a incursionar en actividades tradicionalmente "femeninas", participando más en el hogar y en el cuidado de los hijos, lo que ha exigido también el reacomodo de roles y patrones de comportamiento (Rocha, 2004).

No obstante, lo que se observa es que la cultura tiene su ritmo y que los cambios tardan en gestarse. Ya que, al parecer, todavía se siguen reproduciendo las expectativas de cómo ser mujer y cómo ser hombre, y lo que se espera en cada caso. Así, en el ámbito de la pareja se pueden observar diversas problemáticas dado el doble mensaje, entre lo que la sociocultura dictamina que es lo adecuado y lo que las nuevas generaciones viven en su cotidianeidad.

Por tal motivo consideramos importante investigar si la familia como principal agente socializador influye en los aspectos que las personas consideran importantes a la hora de elegir pareja.

\section{MÉTODO}

\section{Participantes}

Para llevar a cabo el presente estudio se aplicó un cuestionario abierto a 228 participantes: de los cuales 154 eran mujeres (71 con pareja, 83 sin pareja) y 74 hombres (47 con pareja, 27 sin pareja). Las edades fluctuaron entre los 19 y 56 años. Provenían de diversas universidades públicas, privadas y algunos centros de trabajo.

\section{Instrumento}

El cuestionario abierto consistió en las siguientes preguntas: ¿qué cosas o consejos se dan o se han dado en tu familia sobre qué características son deseables para elegir una pareja?; ¿qué otros dichos o consejos se dan en general en la cultura mexicana al respecto de la elección de pareja?, y ¿has cumplido con alguno de los consejos de la familia o de la cultura cuando has elegido pareja?
Se identificaron 28 categorías, las cuales se agruparon por su significación semántica en cuatro familias: 1) aspectos socioculturales: valores, consejos, trabajo, tradicionalismo, respeto, dinero/recursos, clase social/ nivel socioeconómico, sin vicios, proyecto de vida, educación, buena familia, familia similar, sin compromisos, estabilidad/seguridad, religiosidad; 2) interacción con la pareja: que te quiera, bondad, amor, con quien me sienta bien, conocer a la persona; 3) aspectos físicos: atracción/química, edad, apariencia física, y 4) aspectos de personalidad: personalidad similar, inteligencia, madurez/estabilidad emocional.

\section{Procedimiento}

Para llevar a cabo dicho estudio se aplicó el cuestionario abierto a personas de 19 años en adelante que tuvieran por lo menos estudios de preparatoria o su equivalente. La aplicación se realizó principalmente en escuelas y con el método bola de nieve, asegurando el anonimato y confidencialidad de los datos a cada participante. Ya recabada la información, se continuó con la captura de las respuestas. El análisis de contenido se realizó con la ayuda del programa Atlasti5 para identificar códigos y categorías derivados de las teorías antecedentes sobre la elección y el emparejamiento. Para el análisis estadístico se realizaron análisis de frecuencia por sexo y categoría, así como un análisis de $\mathrm{X}^{2}$ con la finalidad de observar las diferencias significativas por sexo y categoría. La descripción de los resultados se presenta a continuación.

\section{RESULTADOS}

Como se observa en la tabla 1 , de todos los códigos analizados sólo algunos mostraron diferencias entre hombres y mujeres. Por ejemplo, en la familia de los aspectos socioculturales los códigos trabajo, que hace referencia a que la pareja trabaje, y dinero/recursos mostraron diferencias significativas principalmente para las mujeres, que refieren es un aspecto que la familia o la cultura recomiendan al elegir una pareja. Lo cual se confirma con lo encontrado por Fisher (2004), quien afirma que en general las mujeres buscarán parejas que puedan proveer los recursos necesarios para la sobrevivencia de la descendencia.

El código tradicionalismo mostró diferencias significativas principalmente para los hombres; éste alude 
a los roles que suelen asignarse a ellos y a las mujeres. En este caso se observa que la cultura, a través de la familia les recomienda elegir parejas tradicionales, es decir, que sepan cocinar, que sean mujeres de casa, que sean buenas madres, etc., así también que sean de buena familia, pues eso hablaría de una mujer de buenas costumbres. En un estudio realizado por Valdés Medina (2008) se encontró que al describir lo que le toca hacer a la mujer mexicana los hombres explicaron que debe ser amiga, comprender, atender el hogar, ser fiel, abnegada y estudiar, es decir, ser buena mujer, sumisa, que cumpla con el rol de buena madre y esposa.
El siguiente código que mostró diferencias significativas, fue el de respeto. En este sentido se tiene que es un factor que se recomienda particularmente para las mujeres. Por otra parte, también se encontraron importantes diferencias, sobre todo en las mujeres, para la recomendación de que la pareja no tenga vicios ni otros compromisos, es decir, que no sea casado o tenga otra familia, pues es muy común encontrar este patrón de la "casa chica", sobre todo en la cultura mexicana. Invariablemente también se recomienda que no tenga ningún vicio, pues esto traerá dificultades a la relación.

Tabla 1. Aspectos socioculturales en la elección de pareja

\begin{tabular}{|c|c|c|c|c|c|}
\hline \multicolumn{6}{|c|}{ Aspectos socioculturales } \\
\hline Código & $\begin{array}{l}\text { Frecuencias } \\
\text { totales }\end{array}$ & $\begin{array}{c}\% \\
\text { Hombres }\end{array}$ & $\begin{array}{c}\% \\
\text { Mujeres }\end{array}$ & $\begin{array}{l}X^{2} \\
\text { sig }\end{array}$ & Ejemplo \\
\hline Valores & 104 & $39 \%$ & $49 \%$ & .140 & $\begin{array}{l}\text { Respeto/responsabilidad, honestidad, } \\
\text { cooperación, solidaridad, tolerancia, humildad, } \\
\text { etc. }\end{array}$ \\
\hline Consejos & 97 & $35 \%$ & $46 \%$ & .087 & $\begin{array}{l}\text { Que la persona te quiera más que tú, que } \\
\text { trabaje, que tenga dinero, etc. }\end{array}$ \\
\hline Trabajo & 90 & $12 \%$ & $53 \%$ & .000 & Que trabaje, que tenga trabajo. \\
\hline Tradicionalismo & 85 & $49 \%$ & $28 \%$ & .007 & $\begin{array}{l}\text { Que sea limpia, responsable, amable, que trate } \\
\text { bien a su madre, que sea responsable, etc. }\end{array}$ \\
\hline Respeto & 62 & $16 \%$ & $32 \%$ & .003 & Ser respetuosa, que nos respetemos, respetarlo. \\
\hline Dinero/recursos & 53 & $15 \%$ & $27 \%$ & .012 & $\begin{array}{l}\text { Que tenga dinero, que tenga que ofrecerte, } \\
\text { que tenga carro. }\end{array}$ \\
\hline $\begin{array}{l}\text { Clase social/nivel } \\
\text { socioeconómico }\end{array}$ & 52 & $16 \%$ & $25 \%$ & .075 & $\begin{array}{l}\text { Buen nivel económico, misma clase social, buen } \\
\text { nivel, etc. }\end{array}$ \\
\hline Sin vicios & 32 & $1 \%$ & $19 \%$ & .000 & Sin vicios, que no se drogue, etc. \\
\hline Proyecto de vida & 23 & $8 \%$ & $11 \%$ & .353 & $\begin{array}{l}\text { Metas a futuro, deseos de superarse, metas en } \\
\text { común, etc. }\end{array}$ \\
\hline Educación & 20 & $7 \%$ & $10 \%$ & .317 & Educado (a), agradable, buenos modales, etc. \\
\hline Buena familia & 17 & $15 \%$ & $5 \%$ & .005 & $\begin{array}{l}\text { Que sea de buena familia, de buenas } \\
\text { costumbres, etc. }\end{array}$ \\
\hline
\end{tabular}




\begin{tabular}{|l|c|c|c|c|l|}
\hline Código & $\begin{array}{c}\text { Frecuencias } \\
\text { totales }\end{array}$ & $\begin{array}{c}\% \\
\text { Hombres }\end{array}$ & $\begin{array}{c}\% \\
\text { Mujeres }\end{array}$ & $\begin{array}{c}\mathbf{X}^{2} \\
\text { sig }\end{array}$ & \multicolumn{1}{|c|}{ Ejemplo } \\
\hline Familia similar & 16 & $5 \%$ & $7 \%$ & .471 & $\begin{array}{l}\text { Que tenga una familia similar, las mismas } \\
\text { costumbres, la misma educación, etc. }\end{array}$ \\
\hline Sin compromisos & 16 & $3 \%$ & $9 \%$ & .018 & $\begin{array}{l}\text { Que no sea casado, sin hijos, sin compromisos } \\
\text { anteriores, etc. }\end{array}$ \\
\hline $\begin{array}{l}\text { Estabilidad/ } \\
\text { seguridad }\end{array}$ & 15 & $5 \%$ & $7 \%$ & .491 & $\begin{array}{l}\text { Que me proteja, que sea estable, que tenga un } \\
\text { buen trabajo, etc. }\end{array}$ \\
\hline Religiosidad & 6 & $1 \%$ & $3 \%$ & .102 & $\begin{array}{l}\text { Que tenga la misma religión, que sea religiosa, } \\
\text { etc. }\end{array}$ \\
\hline
\end{tabular}

Tabla 2. Características sobre la interacción con la pareja

\begin{tabular}{|c|c|c|c|c|c|}
\hline \multicolumn{6}{|c|}{ Interacción con la pareja } \\
\hline Código & $\begin{array}{l}\text { Frecuencias } \\
\text { totales }\end{array}$ & $\begin{array}{c}\% \\
\text { Hombres }\end{array}$ & $\begin{array}{c}\% \\
\text { Mujeres }\end{array}$ & $\begin{array}{l}X^{2} \\
\text { sig }\end{array}$ & Ejemplo \\
\hline Que te quiera & 65 & $23 \%$ & $31 \%$ & .122 & $\begin{array}{l}\text { Que te quiera, que te respete, que te } \\
\text { haga feliz. }\end{array}$ \\
\hline Bondad & 22 & $3 \%$ & $10 \%$ & .007 & $\begin{array}{l}\text { Que sea buena persona, de buenos } \\
\text { sentimientos, amable, etc. }\end{array}$ \\
\hline Amor & 16 & $9 \%$ & $8 \%$ & .695 & $\begin{array}{l}\text { Que me ame, con mucho amor, pareja } \\
\text { romántica, etc. }\end{array}$ \\
\hline $\begin{array}{l}\text { Con quien me } \\
\text { sienta bien }\end{array}$ & 14 & $5 \%$ & $7 \%$ & .491 & $\begin{array}{l}\text { Que me trate bien, que me lleve bien } \\
\text { con la persona, etc. }\end{array}$ \\
\hline $\begin{array}{l}\text { Conocer a la } \\
\text { persona }\end{array}$ & 9 & $7 \%$ & $2 \%$ & .109 & $\begin{array}{l}\text { Tomarse tiempo para conocerse, ser } \\
\text { novios un buen tiempo, etc. }\end{array}$ \\
\hline
\end{tabular}

En el rubro de las recomendaciones de la familia y la cultura en cuanto a la interacción con la pareja se encontró que el código de bondad (ver tabla 2) mostró diferencias significativas para las mujeres, esto hace referencia a la importancia de que la pareja sea una buena persona, pues para sobrellevar una relación se percibe como necesario que esté abierta a la comunicación, sea amable, y de buenos sentimientos. En este sentido, Valdez Medina (2008) encontró que cuando les preguntaba a hombres y a mujeres sobre los aspectos que consideraron para elegir una pareja, ellas mencionaron que buscaban un hombre cariñoso, divertido, compatible, con personalidad, valores y honesto. Los hombres, por su parte, contestaron que buscan mujeres atractivas, comprensivas, que tengan ojos bonitos, sean buenas amigas, bellas y con una bonita forma de ser.

En cuanto a lo deseable o recomendable por la familia y la cultura con respecto a los rasgos físicos (ver tabla 3), se encontró que sólo el código de edad mostró que para las mujeres es importante ese rubro. En este ámbito se ha encontrado que los hombres prefieren mujeres más jóvenes y con mayor diferencia de edad a medida que su edad aumenta; en el caso de ellas, eligen hombres mayores y con menor diferencia de edad en relación con el aumento de su propia edad (Kenrick \& Keefe, 1992). No obstante, en esta investigación la recomendación resultó significativa sólo para las mujeres. 
Tabla 3. Características físicas

\begin{tabular}{|c|c|c|c|c|c|}
\hline \multicolumn{6}{|c|}{ Aspectos físicos } \\
\hline Código & $\begin{array}{l}\text { Frecuencias } \\
\text { totales }\end{array}$ & $\begin{array}{c}\% \\
\text { Hombres }\end{array}$ & $\begin{array}{c}\% \\
\text { Mujeres }\end{array}$ & $\begin{array}{l}X^{2} \\
\text { sig }\end{array}$ & Ejemplo \\
\hline $\begin{array}{l}\text { Atracción/ } \\
\text { química }\end{array}$ & 67 & $26 \%$ & $29 \%$ & .442 & $\begin{array}{l}\text { Que sienta química, que me guste, que } \\
\text { esté bonita-guapo, etc. }\end{array}$ \\
\hline Apariencia & 15 & $4 \%$ & $8 \%$ & .157 & $\begin{array}{l}\text { Que vista bien, que dé buena impresión, } \\
\text { de buen ver, etc. }\end{array}$ \\
\hline Edad & 9 & $1 \%$ & $5 \%$ & .058 & $\begin{array}{l}\text { Más grande que yo, más chica que yo, de } \\
\text { mi edad, etc. }\end{array}$ \\
\hline
\end{tabular}

Por otro lado, en la familia de los aspectos de personalidad, el código de personalidad similar (ver tabla 4) mostró una diferencia significativa para los hombres, ya que al parecer la recomendación es que elijan una pareja con gustos afines, intereses comunes y compartan gustos y costumbres. En un estudio que planteaba la cuestión de ¿cómo eligieron las personas a sus parejas?, García Meraz y Reyes Lagunes (2008) encontraron que la personalidad se concebía como: que la persona sea buena onda, tenga cualidades, con calidad humana, de buenos sentimientos, noble, de buenos hábitos, de buen temperamento y con determinada forma de ser. A su vez, también la similitud fue un elemento mencionado para elegir pareja, refiriendo que lo ideal es que ésta sea similar en pensamientos, características y actitudes.
Finalmente, los resultados obtenidos muestran que el $65.78 \%$ de la muestra encuestada dijo que sí cumple con lo aconsejado por la familia y la cultura; los aspectos que más se mencionan en este sentido son: los valores, que sea una persona que te quiera, que tenga una personalidad similar y en el proyecto de vida.

\section{DISCUSIÓN}

La elección de un compañero o compañera es uno de los problemas más importantes e interesantes que se plantea la psicología de las relaciones interpersonales. Aparentemente, sucede casi al azar. Numerosos sexólogos, biólogos, antropólogos y psicólogos se han esforzado por establecer, desde el punto de vista científico, las motivaciones de la elección amorosa. Las cualidades físicas, la belleza, la fuerza corporal, los ojos, la nariz,

Tabla 4. Aspectos de personalidad

\begin{tabular}{|l|c|c|c|c|l|}
\hline \multicolumn{1}{|l|}{ Aspectos de personalidad } \\
\hline Código & $\begin{array}{c}\text { Frecuencias } \\
\text { totales }\end{array}$ & $\begin{array}{c}\% \\
\text { Hombres }\end{array}$ & $\begin{array}{c}\% \\
\text { Mujeres }\end{array}$ & $\begin{array}{c}\mathbf{X}^{2} \\
\text { sig }\end{array}$ & \multicolumn{1}{|c|}{ Ejemplo } \\
\hline $\begin{array}{l}\text { Personalidad } \\
\text { similar }\end{array}$ & 36 & $22 \%$ & $12 \%$ & .048 & $\begin{array}{l}\text { Que comparta gustos, mismas } \\
\text { costumbres, intereses comunes, etc. }\end{array}$ \\
\hline Inteligencia & 33 & $14 \%$ & $15 \%$ & .647 & Que sea inteligente. \\
\hline $\begin{array}{l}\text { Madurez/ } \\
\text { estabilidad } \\
\text { emocional }\end{array}$ & 15 & $5 \%$ & $7 \%$ & .491 & $\begin{array}{l}\text { Que sea maduro emocionalmente, que } \\
\text { sea estable, etc. }\end{array}$ \\
\hline
\end{tabular}


la boca, los cabellos, las piernas, las manos y todas las partes del cuerpo son susceptibles de atraer la atención, consciente o inconscientemente, sobre cualquier persona que se encuentra por casualidad.

Sin embargo, la investigación en este ámbito ha demostrado que surgen cada vez más aristas al tratar de definir cuáles son los aspectos implicados en el fenómeno de la elección y el emparejamiento.

Así que la elección de pareja se da en primera instancia por la atracción de dos personas, aunque posteriormente intervendrán una serie de factores que facilitarán o entorpecerán el establecimiento de una relación diádica. En este punto, la investigación ha encontrado que la cercanía es un factor determinante en el emparejamiento (Brehm, Miller, Perlman \& Campbell 2007). Otros estudios muestran que nos parecen atractivas las personas con una personalidad similar a la nuestra (Blankenship, Hnat, Hess \& Brown, 1984). También se ha encontrado que nos emparejamos con personas similares a nosotros en atractivo físico (Dion, Berscheid \& Walster, 1972). Entre muchas otras cosas más, elegimos pareja con base en una diversidad de elementos; sin embargo, como se puede observar en esta investigación, la familia es una de las principales trasmisoras de información sobre lo deseable al elegir pareja, ya que es a través de ésta que se trasmiten los patrones de endoculturación, reglas, normas, usos y costumbres, formas de ser y los roles esperados para cada sexo en función de lo que dicta la cultura (Díaz Guerrero, 2007). Este estudio evidencia como en el ámbito de las relaciones interpersonales y de pareja, no necesariamente se hace una elección personal, parece que se escoge en función de lo que los padres sugieren es lo adecuado. Explícita o implícitamente se sabe a través de consejos, mandatos o conductas que será aceptado en ese grupo familiar.

No obstante, en el presente estudio se encontró que la familia tiene influencia implícita o explícita en cuanto a lo que es deseable que sus miembros elijan como pareja. Tal como lo refiere Quilodrán (2001), en la familia mexicana se siguen los patrones de la familia de origen y extensa (abuelos, padres, tíos y otros familiares), que ejerce gran influencia sobre los miembros de la pareja y el desarrollo de su relación.

Probablemente sea el grupo familiar de origen el que orienta esta elección. No es que el casamiento se decida de manera autoritaria, sino que existen presiones más sutiles que siguen desempeñando su papel, aun cuando el individuo ha logrado su autonomía.

En este sentido, Pérez, Estrada y Pacheco (2007) refieren que la pareja de origen (los padres o los cuidadores primarios) y los principios morales (aspectos socioculturales inmediatos) influyen en las conductas y creencias que posee cada género, lo cual contribuye a la generación de expectativas hacia la pareja. Así también explican que dichas expectativas pueden facilitar o determinar las características del vínculo, lo cual influirá en el proyecto de vida de la pareja y en los cambios personales.

Como se muestra en el presente estudio, más de la mitad de los participantes dice tomar en cuenta lo que le aconseja su familia con respecto a la elección de la pareja, sobre todo en lo que se refiere a los valores, pues se espera que ésta sea una persona honesta, responsable y respetuosa, lo cual puede hablar de una persona confiable con la cual establecer una relación con mayor compromiso. Así también se encontró que la premisa popular, o casi un mandato sociocultural es "que busquemos parejas que nos quieran más que nosotros a ellas", ya que de alguna manera esta premisa asegura una relación más duradera, pues el amor y el afecto recibido harán que la potencial pareja permanezca en la relación. Sin embargo, esto no necesariamente opera así en la realidad.

Aunado a lo anterior, se tiene que también es recomendación de la familia y la cultura que se elija una pareja similar en personalidad y que a su vez se tengan proyectos de vida afines, ya que esto asegurará de alguna forma que las diferencias no sean tan grandes y que en la semejanza se pueda coincidir en los acuerdos y proyectos que se planteen a futuro en la relación.

En este sentido, las interacciones entre individuos en un vínculo de pareja estarán mediadas por la relación con el contexto sociocultural en el que están insertas. Al respecto, Sternberg (2000) afirma que los antiguos estereotipos sociales no cambian tan fácilmente. Lo cual nos lleva a la consideración respecto a cómo ciertas, normas, reglas, premisas y patrones conductuales permanecen inertes a través de las generaciones (Díaz Guerrero, 2007); finalmente seguimos siendo el reflejo de lo que sucede en la familia y por ende ésta es el reflejo de una sociedad que, a pesar de sus múltiples 
transformaciones, en sus premisas el proceso es mucho más lento.

Por ejemplo, es interesante observar que en el código tradicionalismo que refiere a los roles que suelen ser asignados por costumbre a cada sexo, los hombres que participaron en este estudio indican ser aconsejados por sus familias para que elijan una mujer tradicional, es decir, que se dedique al hogar, sea ama de casa, cuide a los hijos, sea limpia, maternal, amable, etc. Así, al parecer las familias siguen reproduciendo la tendencia a fomentar los hogares tradicionales, en donde el hombre es ajeno a las actividades del hogar, a la crianza de los hijos, así como a compartir una relación de pareja más equitativa y una paternidad responsable.

Esto también se puede observar en la premisa aconsejada sobre elegir a una pareja de "buena familia", entendida como una familia de buenas costumbres, honesta, de buenos sentimientos, bondadosa, etc., tal vez pensando en las relaciones familiares que se establecerán a futuro, en cuanto la nueva pareja se consolide. Lo cual nos refiere a la premisa del status quo

\section{REFERENCIAS}

Blankenship, V., Hnat, S. M., Hess, T. G., \& Brown, D. R. (1984). Reciprocal interaction and similarity of personality attributes. Journal of Social and Personal Relationships, 1, 415-432.

Brehm, S., Miller, R W., Perlman, D.S. \& Campbell, S. M. (2007). Intimate relationships. (4a. Ed.). Nueva York: McGraw-Hill Higher Education.

Buss, D. \& Schmitt, D. P. (1993). Sexual strategies theory: an evolutionary perspective on human matting. Psychological Review, 100, 204-232.

Díaz Guerrero, R. (2003). Psicología del mexicano. (6a. reimpresión). México: Trillas.

Díaz Guerrero, R. (2007). Psicología del mexicano 2. Bajo las garras de la cultura. México: Trillas.

Díaz-Loving R., Rocha Sánchez, T. E. \& Rivera Aragón, S. (2007). La instrumentalidad y la expresividad desde una perspectiva psico-socio-cultural. México: Porrúa.

Dion, K. K., Berscheid, E. \& Walster, E. (1972). What is beautiful is good. Journal of Personality and Social Psychology, 2, 285-290. familiar que ya Díaz Guerrero (2003) propuso como una forma de mantener las jerarquías y las alianzas familiares.

Por otra parte, las recomendaciones para las mujeres más representativas son que la potencial pareja sea trabajadora y que posea cierta estabilidad en cuanto a lo económico y el poder adquisitivo, pues esto asegurará que, de conformarse una familia, la descendencia tendrá acceso a lo necesario. Lo cual reitera lo referido en la literatura (Buss \& Schmitt, 1993) en donde las mujeres buscarán parejas que posean estabilidad económica, pues esto es señal de seguridad, protección y apoyo en una relación a largo plazo.

A manera de conclusión, este estudio contribuye al estudio del emparejamiento y la elección de pareja, resaltando la importancia de que por lo menos en la cultura mexicana aun sigue influyendo la familia, y que por tanto, para estudios subsecuentes, es importante considerar este rubro, ya que la elección se torna un fenómeno muy complejo que debe ser estudiado en sus diversos componentes y elementos intervinientes.

Fisher, H. (2004). Por qué amamos. Naturaleza y química del amor romántico. México: Taurus.

Gangestad, S. W., Haselton, M. G. \& Buss, D. (2006). Evolutionary foundations of cultural variation: Evoked culture and mate preferences. Psychologican Inquiry, 17(2), 75-95.

García Meraz, M. \& Reyes Lagunes, I. (2008). Construcción y validación de un instrumento de elección de pareja. En R. Aragón, R. Díaz-Loving, R. Sánchez y I. Reyes (Eds.). La psicología social en México. (Vol. XII. pp. 599- 604). México: Amepso.

Garrido Garduño, A., Reyes Luna, A. G., Torres Velázquez, L. E. \& Ortega Silva, P. (2008). Importancia de las expectativas de pareja en la dinámica familiar. Enseñanza e Investigación en Psicología, 13 (2), 231-238.

Giddens, A. (2001). La transformación de la intimidad: sexualidad, amor y erotismo en las sociedades modernas. España: Cátedra.

Kenrick, D. T. \& Keefe, R. C. (1992). Age preference in mates reflects sex differences in human reproductive strategies. Behavioral and Brain Sciences, 15, 75-133. 
Pérez Aranda, G., Estrada Carmona, S. \& Pacheco Quijano, L. V. (2007). Iguales y diferentes: Análisis cualitativo de las vivencias de hombres y mujeres sobre su relación de pareja. Archivos Hispanoamericanos de Sexología, XIII(2), 169-192.

Quilodrán Salgado, J. (2001). Un siglo de matrimonio en México. México: El Colegio de México. Centro de Estudios Demográficos y de Desarrollo Urbano.

Quilodrán Salgado, J. \& Sosa Márquez, V. (2004). El emparejamiento conyugal: una dimensión poco estudiada de la formación de las parejas. En M. Ariza y O. Oliveira (2004). (Eds.) Imágenes de la familia en el cambio de siglo. México: Universidad Nacional Autónoma de México. Instituto de Investigaciones Sociales.

Rocha, S. T. \& Díaz-Loving, R. (2004). Desarrollo y validación de una escala de estereotipos de género. En R. Aragón, R. Díaz-Loving, R. Sánchez y I. Reyes (Eds.).
La psicología social en México, 10, 41-48. México: Amepso.

Sternberg, R. (2000). La experiencia del amor. España: Paidós.

Trivers, R. L. (1972). Parental investment and sexual selection. En B. Campbell (Ed.), Sexual selection and the descent of man: 1871-1971. (136-179). Chicago: Aldine.

Valdés Medina, J. L., González Arratia, N. I. \& Sánchez Valdovinos, P. (2005). Elección de pareja en universitarios mexicanos. Enseñanza e Investigación en Psicología, 10(002) 355-367. Xalapa: Universidad Veracruzana.

Valdez Medina, J. L. (2008). Los roles contemporáneos de los hombres y las mujeres en México. En R. Díaz-Loving (compilador). Etnopsicología mexicana. Siguiendo la huella teórica y empírica de Díaz Guerrero. México: Trillas. 